\title{
ALI-MENTI: DONNA E ALIMENTI SURREALI
}

\author{
ALI-MENT: WOMAN AND SURREALISTIC ALIMENT
}

Ludovica Radif

Università Palacký Olomouc

\section{Riassunto:}

La ballerina, protagonista dei racconti, insieme al Manichino, suo compagno di viaggio, si sottrae alla scena propriamente detta specialmente attraverso il cibo, sia per la scelta dei locali che fanno da sfondo, sia per la selezione degli alimenti, che sembrano smaterializzarsi in "ali" e in "menti", ossia in evasione, sogno e cultura. E il suo diario, origine del racconto, sconfina oltre le quinte...

\section{Parole Chiave}

Manichino a tavola; ali-menti ed evasione; cibo neosurrealista; oggetto neosurrealista

\section{Abstract:}

Together with her Mannequin, the bright dancer, protagonist of these surrealistic tales, is travelling around the world, visiting in artistic cities, but disappears, it seems, from the scene, especially through the food, the "aliment", which becomes alate-mental occasions for dreaming and culture in designed atmospheres. And her diary, source of tales, exceeds the limits of the scene...

\section{Keywords:}

Neo-Surrealistic food; Mannequin, dancer and aliments; table and dreaming 
Il cibo come fatto culturale, come riflesso di una comunità, è un dato ormai acquisito e continuamente studiato in riviste specialistiche e non, sotto innumerevoli aspetti, da quello più squisitamente antropologico a quello più sottilmente poetico-letterario. Per esempio, ne sono stati messi in evidenza i risvolti culturali da Massimo Montanari in svariati contributi (Montanari 2004, 2008, 2013), a partire specialmente dall'epoca medievale. Più di recente ne è stato considerato il ruolo nell'ambito dell'emigrazione come fenomeno interessante tra mantenimento di identità e ricerca di socializzazione e integrazione (cfr. sull'argomento il testo Pravettoni 2015). La rilevanza sociale del tema si può constatare anche nella fortuna riscontrata dai convegni e manifestazioni fieristiche ad esso dedicati (come l'Expo, per cui si può consultare la sintesi di Dansero, Egidio-Giorda, Mariachiara-Pettenati Giacomo 2015) che ne assemblano due aspetti come fondamentali e a volte persino speculari di un popolo: il cibo per il corpo e il cibo per lo spirito. Il rapporto tra l'alimentazione e la surrealtà, che può anche scaturire dalla cultura, non risulta invece ancora molto esplorato, se non a sprazzi a livello di sperimentazione artistica (per esempio, la mostra "Improbabilità" di Giuseppe Colarusso che propone oggetti quotidiani, anche elementi commestibili, in situazioni e combinazioni non reali).

Dal momento che l'apparecchiarsi di una tavola surreale può divenire un trampolino oltre la scena e quindi portare i personaggi che lo assaggiano, nello specifico la donna, al di là delle quinte, pensiamo che possa essere interessante presentare in questa sede il cibo surreale come viene vissuto e descritto nella Tetralogia del Teatro del Manichino (Radif 2012a, 2012b, 2013, 2016).

La tavola, che assume i contorni quasi di una dimensione fiabesca o leggendaria, comunque a qualche metro da terra, è conseguenza anzitutto di una particolare modalità di osservazione; il menù presentato nei racconti viene scelto dai protagonisti non tanto, come solitamente avviene per gli uomini, sulla base del gusto, ma per esempio per il suo aspetto o per il suono.

Consideriamo in proposito il piatto del risotto ai carciofi, così come viene descritto nel primo racconto della serie (pp. 24-25). La ragazza è convinta che una vita al fianco del Manichino le riservi una ricchezza inesauribile, quella del saper vedere in modo artistico le situazioni attorno. E in effetti il pasto consumato dai due a casa viaggia su una siffatta lunghezza d'onde. Il risotto può allora trasformarsi in un riso, in senso comico, una sagoma comica, così come il carciofo, erede di una tradizione araba, ritrova la sua natura di fiore alternativo, da invasare, così come invasati sembrano loro due a scherzarvi intorno. Analogamente il dopocena si fa arte in un quadro, capace di far viaggiare le menti per i suoi toni orientali (abbondano le spezie, come un riflesso gastronomico della dimensione orientaleggiante e nomade dei loro incontri). 
Ecco dunque che la casa che li ospita a cena perde i connotati reali, facendo elevare i protagonisti in una dimensione altra, dove a dominare è la suggestione dell'antico e del sorriso. Di riflesso notiamo una curiosa duttilità delle parole, che si piegano per rendere la novità semantica dei loro contenuti nel contesto: infatti "risotto" non esiste in italiano come termine riferito alla dimensione comica, ma si presta qui a indicare un riso improvvisato o abbozzato; anche l'etimologia del carciofo viene riscoperta nella funzione arabeggiante di cui si intende ammantare la scena. Nella scena clou, che dà il titolo al racconto, ritroviamo un oggetto casalingo in un ruolo poetico simbolico: sullo sfondo omerico di un'aurora "dalle dita di rosa" che si monetizza in una caparra di felicità, domina infatti la torretta di una promettente caffettiera, prossima a riversare sui due, sentinelle della loro stessa impresa, l'aromatica colazione. Come spesso si riscontra nei racconti, l'istante della sospensione, l'attesa casuale in cui tante situazioni anche insignificanti si frammentano, diventa occasione illuminante per il tempo a seguire. È proprio a fornello acceso che i due si peritano in un tango; il dettaglio permette un balzo in avanti o indietro al quel momento in cui nel terzo racconto, all'archivio, la protagonista estrae la tessera del corso di tango. Evidentemente dunque la sua capacità ha incuriosito il Manichino, che ha così desiderato cimentarsi in due tre passi, brevi, ma così intensi da prospettare l'ipotesi di un futuro.

Tra gli espedienti linguistici impiegati per la trasformazione di una scena reale in un contesto surreale contiamo il neologismo surrealista e la voluta deformazione delle parole, con effetto di slittamento. Il lettore crede di aver compreso cosa sta succedendo e invece rimane impigliato mentalmente in qualche non-sense o in un senso inaspettato che lo conduce verso un altro ordine semantico. Possono essere l'etimo o le possibili implicature del termine a mettere in evidenza l'aspetto che pertiene alla storia. Anche il processo di ri-semantizzazione coinvolge l'espressione del racconto. Quando i due scendono nella dispensa si parla di una katàbasi, un'impresa che ha un sapore storicoepico ("discesero adunque l'oscura dispensa/oltre la barriera dei detersivi/alla baia salmastra di minestre e affini") (Radif, 2012: 25).

Anche l'oblò che essi varcano inscrive la scena in una possibile ambientazione fantascientifica, ma il colore giallo lo smorza in toni più giocosi; all' interno, nello spazio relativo alle provviste, abbiamo la baia salmastra, qualcosa di marino-balneare che viene però immediatamente corretto come "baia salmastra di minestre e affini", quindi trasferendo l'icona balneare in una sorta di oasi del gusto. Essi dunque convertono l'approvvigionamento da un armadio domestico in una sorta di esplorazione di una spiaggia aromatica, per così dire. Nel secondo racconto è un esemplare di cacciagione a imporsi sulla scena, in modo insolito: una faraona, che, nella sua aggettivazione a trencadís, si propone come pezzo d'arte, non solo ma d'arte tipica locale; inoltre, la sua presenza fra gli oggetti di un'asta la impreziosisce del valore aggiunto di rarità. La 
storia è appena iniziata, per cui si può dire il primo oggetto significativo che richiama il lettore per la sua particolarità e sembra infatti contenere qualcosa di misterioso. Su quello il battitore si interrompe, permettendo poi l'agnitio con il Manichino. In una composizione ad anello, che richiama poi l'intera struttura circolare della saga, ritroviamo la faraona verso la fine, quando torna in ballo la questione dell'asta con relativo ufficiale incaricato di riprendersi indietro il manufatto. Qui il nome "faraona" è associato a tutt'altra situazione: è proprio l'animale cucinato e proposto in un piatto. Appositamente, per disorientare e riorientare insieme il lettore verso nuove interpretazioni, si ripropone la domanda iniziale; la confusione reale e mentale che si crea diviene l'occasione da cogliere al volo per la protagonista, che decide così di tenersi il suo speciale "sagomato". Non è inutile forse richiamare anche qui l'attenzione sulla scelta di un piatto che anche nel suo nome esotico sembra adatto a condurre le menti nelle lande egiziane, in un clima di ricercatezze e prelibatezze. Ma è pur vero che i due non stanno mangiando: la faraona è un oggetto distraente che si frappone entro le maglie della quotidianità, permettendo l'inserimento di nuove logiche, come quelle avanzate dalla protagonista. I piatti possono diventare la traduzione di un modo di essere, come quando i due si trovano alla Taverna della Gru; una serie di possibili riferimenti alla favolistica sembrano guidarci entro una situazione alternativa; in effetti tutti i particolari portano al di là della stessa. Mentre la serata si stende come un film attorno al locale, immerso nella calma straniante estiva, al centro assistiamo al dialogo delle portate. I piatti esprimono, quasi naturalmente, il modo di essere dei due, mentre si personifica un gamberetto, in un contesto in cui le cose si animano. La frase finale riassume indirettamente la densità del dialogo di quella sera, dovuto al fatto che tutto intorno ne partecipa e vi partecipa. La serata diviene la regista, da tempo affacciata sul palco ad aspettare i due recitare, mentre la gru, con il suo svettare dalla soglia, pare voler indicare una visione con altra prospettiva. Che il cibo possa diventare un modo per contraddistinguere le diverse personalità si può constatare dal fatto che M., di per sé un oggetto, quindi non bisognoso di nutrirsi, si rivela facilmente coinvolgibile specie in tema di dolciumi (un esempio nel secondo racconto è il salmastro gelato metafisico, p. 8); in effetti impiega anche varie esclamazioni di ambito onomatopeico come slurp. Mentre M. nel gironzolare per le stradine sembra propenso a gustare i vari cibi, Lei appare orientata verso elementi secondari e accessori; per esempio di una birra si pongono in evidenza soltanto i tappi, lasciati su una scacchiera sperando possano portare avanti il gioco da soli.

Nel terzo racconto ci si imbatte nei churros (pp. 31-32); il termine più o meno indirettamente riconduce il lettore della saga al racconto precedente sviluppato sulla scena di Barcellona. I cibi possono diventare quindi occasioni per far partecipe il lettore di una saga in evoluzione, le cui tappe possono riepilogarsi come parti di un unico processo di avventura a capitoli intercambiabili nella cronologia. Caso molto 
particolare sarà la libreria dove i due pervengono nel IV racconto. Gli alimenti sono un richiamo, per chi lo sa cogliere, al passato e al futuro dei protagonisti, portando fuori dalla scena Lei che se ne fa interprete per conto di entrambi.

In modo particolare all'interno della Casa Danzante di Praga si esplicitano anche alcuni passaggi della trasformazione del fatto alimentare in fatto surreale: la presenza dei due all'interno della casa è prima di tutto un'esclusiva, un privilegio rispetto al mondo che ne rimane fuori, come irrimediabilmente al di fuori della loro complicità, alle macchine che sfrecciano a fianco del ponte illuminato. Le esclusive continuano: mentre M. sembra godersi la sua bevanda, Lei ne sposta l'attenzione attraverso una domanda insolita su come sia quell'acqua nel tronco di cono; più che il domandarsi del sapore dell'acqua (domanda già di per sé anomala) il punto a suscitare curiosità è il "tronco di cono"; chi legge inizia a portare gli occhi immaginari su recipienti inconsueti, per poi ritornarli all'indirizzo della protagonista: il tronco di cono non è che un bicchiere. È negli occhi di Lei che il bicchiere assume quel nome, ma la stranezza che ne consegue fa veramente sospettare un che di alchemico. A quella parola si passa dal piano gastronomico al piano magico, dove lui si sente sulla stessa lunghezza d'onda, stando addirittura al gioco e proponendo la tazzina del caffè come "tronco di piramide". Il caffè d'altra parte si percepisce solamente nella schiuma e si istituisce immediato un parallelo con le bolle dell'arredamento modernista della casa al piano terra. Il contesto è quello di una sorta di focolare riparato dove "lucrezianamente" si apprezza la protezione rispetto alle tempeste esterne; partecipa dell'orizzonte anche la musica, che per l'occasione scende in forma di gocce.

Nello stesso contesto, ripreso curiosamente, ma per un motivo ben preciso, nel racconto più recente $A l$ Bolshoi col Manichino, condotto in chiave musicale sulla scena teatrale russa, ritroviamo i protagonisti, intenti a sorbire gustose bollicine spettatrici di un magico momento di confidenze, profondo e onirico al contempo, fuori e dentro la scena, che porta con sé il senso di altre scene percorse.

Ella quando può sceglie con gusto i locali in cui entrare, che faranno da sfondo al suo piacevole intrattenersi col Manichino, prende il menù (anche se, nel caso del Foyer dell'elfo smeraldo, ammette, preferirebbe scegliere la sua consumazione leggendo uno spartito musicale), e si lascia attirare da qualche piatto, il titolo, il contorno, un dettaglio... Ma le motivazioni spesso trascendono, per così dire, gli ingredienti: può essere il nome esotico, o una giocosa coincidenza a dettargliela; al momento in cui si deposita sul tavolo, il cibo assume spesso una veste anomala, per come viene visto e poi vissuto dai personaggi come qualcosa di altro. A volte passa completamente in secondo piano, divenendo sostanzialmente un mero pretesto per la conversazione, quasi uno spunto di dialogo esso stesso; altre volte rimane in un angolo a raffreddarsi, perché altri interessi incombono e portano altrove la mente. 
Il cibo diviene cultura, non solo perché riguardato nella sua ricetta, e spesso si riporta il nome straniero, proprio per invitare il lettore a entrare il più possibile nell'atmosfera del racconto di viaggio, ma anche perché permette di ritagliarsi un'occasione di dialogo. Ecco perché, con un gioco di parole, si è voluta scrivere la parola "ali-menti" come fosse un termine composto di "ali" e "menti": le "menti" emergono dagli alimenti, entrano in dialogo con la cucina, con le ricette, con le personalizzazioni delle stesse, con chi le gusterà.

Vediamo ora l'altra parte del bizzarro composto: le ali.

Nel racconto del Bolshoi fa da sfondo ai loro incontri in teatro il Mefistofele di Arrigo Boito, e i cori degli angeli sono riecheggiati attraverso versetti ingenui di osanne (si pensi al "fruscio d'osannanti"), danze, luci e colori celesti: dell'affare dell'anima di Faust rimangono soprattutto, anche se in una chiave fresca, non impegnata, leggera come il surreale di suo implica, gli aneliti alla trascendenza, che alla fine risulta trionfante.

Anche la tavola dei due protagonisti non è esente da viva e vivace aspirazione verso l'altro e l'alto, verso il meglio, e se il Manichino sta sempre un passo avanti, in quanto rappresenta il sogno da rincorrere, è pur vero che la tavola... vola, quando la si viva con un certo spirito.

Ecco perché i piatti solitamente non hanno l'aspetto corposo della lasagna al forno, qualcosa di statico e consistente, ma un'identità più versatile, come le bibite frizzanti che si volatilizzano attorno, o le zuppe piccanti, che sembrano quasi sciogliersi in bocca durante la conversazione.

La ballerina che viaggia con il suo Manichino potrebbe cibarsi di arte, saltare i pasti, ignorando un bisogno fisico che potrebbe troppo ancorarla alla terra, impedendole il volo. Oppure, infilarsi in un ristorante e consumare quel pasto che nulla toglie alla nobiltà del suo essere viaggiatrice. Ma così non è e, se non segue diete, non ha neppure molta fame: e come la vediamo, nella serie dei racconti del Manichino, ricercare in teatro il bagno per signore, così, golosa più che affamata, entra abitualmente in graziosi bistrot dove rifocillarsi, dove è possibile consultare un menù, pranzare, cenare, forse anche danzare. Eppure, che sia da sola o in compagnia, il suo modo di sostare è presenza e assenza insieme rispetto al contesto, assaggio che pare piuttosto un fatto dell'anima rispetto alla forchetta. Sfuma via dal contesto, come non vi fosse dentro, ma ne carpisce dettagli come lo fotografasse. E non è una visione virtuale, asettica, da lontano, da report di estranei, tutt'altro, sembra coglierne la sostanza. Insomma è la tavola surreale quella che si apparecchia a Lei, e indirettamente ai suoi lettori.

Qualcosa di vero, qualcosa di fiabesco nei nomi dei locali crea in chi legge un effetto di straniamento come per una lente trasferita troppo rapidamente per lasciar vedere la 
realtà così com'è; ed è invece per come Lei vede, descrive, vive, in quanto interprete dei luoghi e dei toni, che il lettore è accolto all'interno dello spazio narrativo.

Lo slittamento tipico dello stream of consciousness potrebbe trasferire una parete di maioliche di Siviglia sulla superficie a trencadís della Barcellona di Gaudi, come, al contrario, rivivere su una chiesa ortodossa la sequenza artistica delle tessere policrome... Ma se noi provassimo a immaginare la protagonista senza il Manichino dove finirebbe il surreale? Anche quando i due prendono direzioni indipendenti in una giornata, assistiamo a una sincronia di viaggio che li fa sentire comunque vicini e compartecipi di quanto si vede attorno.

I protagonisti entrano in molti ristoranti e sembrano i soli avventori: com'è possibile? Chi c'è intorno? Non c'è nessuno o sono loro a non esser visti o sono loro a non aver occhi che per se stessi? In effetti non sono invisibili agli altri se possono fare ordinazioni ai camerieri, eppure chiunque si avvicini e interagisca con loro sembra rapito in una dimensione altra che rende anche lui un po' anomalo.

Una funzione fondamentale che il mangiare assolve all'interno dei racconti è la trasmissione interculturale attraverso un trapianto linguistico: spesso le lingue di mescolano e intrecciano; cibo, dunque, come assaggio mnemonico, scampolo di viaggio, ponte interculturale. In analogia con il vestiario della ragazza, si seleziona come originale, non corrente, traduce sul fornello la personalità un po' eccentrica; inoltre è chiaro anche il temperamento deciso e ricco di passione, per cui si nominano spesso le spezie, peperoncino e pepe. E dunque diviene la lingua silenziosa con cui si parla a tavola: tinte forti come sapori forti dipingono la cucina dei nostri protagonisti intenti a rifocillarsi durante il viaggio.

Ma che tipo di alimenti prediligono?

Il Manichino ammette di avere una discreta fametta, da potersi mangiare un cinghiale; tra l'altro, incuriosisce e potrebbe essere indagato come, essendo lui in partenza un oggetto, utilizzi a sua volta oggetti e cibi; per Lei lo stomaco parla un linguaggio più sottile. Sembra che la tappa al ristorante sia piuttosto un pretesto per stare vicina, o più vicina a lui, e il menu sia un modo privilegiato per conoscere i suoi gusti, una forma di conoscenza molto efficace, alternativa a quella verbale.

Se ricorrenti sono le zuppe, ricercate per sapienza di elaborazione, rarità degli ingredienti (per esempio la smetana) e per storicità di tradizione, non mancano i funghi, che permettono in una battuta scherzosa del M., la divagazione sulle presunte proprietà allucinogene di alcune specie. Solitamente sono alimenti sfiziosi, più che nutrienti, bevande (prevale il caffè, anche accompagnato dalla panna); l'impressione del lettore è che si tratti di qualche antipasto da poter assaggiare senza troppo distogliere l'attenzione dal dialogo: lo vediamo nel caso del ristorante del lago, che viene descritto 
proprio come la scena di un teatro rimasta vuota per fare spazio a uno spettacolo tutto dedicato a loro. Ogni alimento citato può essere l'occasione per una storia, una curiosa leggenda reperita sulla guida turistica, piuttosto che per un aggancio o doppio senso rispetto a quello che si evince a prima vista dal dialogo. E in effetti alla fine si dice che quella cena saziava "dentro" la protagonista, era cioè tutto un'imbeversi interiore mentre scorrevano un po' distrattamente davanti a loro gli alimenti (pp. 34-36).

La ballerina grazie a un certo intuito o sesto senso per location "gravide" di mitologia, vi fa ingresso e invita chi le è accanto a partecipare di speciali ambientazioni; con istintiva predilezione per cibi evocativi, "colti", suscettibili di commento, sembra tuttavia creare attorno a sé un ponte di evasione dalla scena stessa, sul quale invita piacevolmente chi, come M., può seguirla nei ragionamenti, o, come l'uomo misterioso che compare di tanto in tanto con frasi rivelatorie, addirittura anticiparne i movimenti.

Gli alimenti sulla tavola dei due protagonisti dunque parlano, trasmettono la cultura di cui sono frutto, esprimono la lingua in cui si traducono, ricordano, concretizzano in un fatto materiale e fisiologico il concentrato di una memoria.

Gli alimenti volano sulle ali dei ricordi, o come trampolini verso un altrove condiviso o ancora soltanto sognato; balzano oltre il piatto, debordano dai bicchieri, filtrano tra i rebbi delle forchette, si riflettono sulle superfici cristalline dei calici e svaporando in bollicine sembrano creare già nell'aria intorno l'anticipazione dei loro progetti.

\section{RifERimenti Bibliografici}

Dansero, E.-G., Mariachiara-Pettenati G., "Per una geografia culturale del cibo", http://frida.unito.it/wn_media/uploads/cicuageo_1432807888.pdf

Radif, L., “Tango col Manichino”, Genova, Compagnia dei Librai, 2012

Radif, L., "Pipistrelli a Batlló. A Barcellona col Manichino”, Genova, Compagnia dei

Librai, 2012

Radif, L., “Alla Casa Danzante col Manichino”, Genova, Compagnia dei Librai, 2013

Radif, L., “Al Bolshoi col Manichino”, Milano, Ledizioni, 2016

Montanari, M, "Il cibo come cultura", Roma-Bari, Laterza, 2004

Montanari, M., "Alimentazione e cultura nel Medioevo", Roma-Bari, Laterza, 2008

Montanari, M., “L'identità italiana in cucina”, Roma-Bari, Laterza, 2013

Pravettoni, R., "Il cibo come elemento di identità culturale nel processo migratorio", Internet. http://docplayer.it/1302690-Il-cibo-come-elemento-di-identita-culturalenel- processo-migratorio.html 